\title{
Association Between Serum Furin and Fasting Glucose: A Cross- Sectional Study in Chinese Adults
}

\author{
Yan $\mathrm{He}^{1 \dagger}$, Hanyun $\mathrm{Zhu}^{2 \dagger}{ }^{\text {, }}$ Min Zhang ${ }^{3 \dagger}$, Jing $\mathrm{Li}^{1}$, Shengqi $\mathrm{Ma}^{2}$, Yin $\mathrm{Lu}^{1}$, Linan Chen ${ }^{1}$, \\ Mingzhi Zhang ${ }^{2 *}$ and Hao Peng ${ }^{1,4 *}$ \\ ${ }^{1}$ Department of Epidemiology, School of Public Health, Medical College of Soochow University, Suzhou, China, \\ ${ }^{2}$ Department of Biostatistics, School of Public Health, Medical College of Soochow University, Suzhou, China, \\ ${ }^{3}$ Central Office, Suzhou National New and Hi-Tech Industrial Development Zone Center for Disease Control and Prevention, \\ Suzhou, China, 4 Jiangsu Key Laboratory of Preventive and Translational Medicine for Geriatric Diseases, Suzhou, China
}

OPEN ACCESS

Edited by:

Tao Huang,

Peking University, China

Reviewed by:

Eusebio Chiefari,

University Magna Graecia of

Catanzaro, Italy

Marco Arieli Herrera-Valdez,

Universidad Nacional Autónoma de

México, Mexico

${ }^{*}$ Correspondence:

Hao Peng

penghao@suda.edu.cn

Mingzhi Zhang

zhangmingzhi@suda.edu.cn

${ }^{t}$ These authors have contributed equally to this work and share

first authorship

Specialty section: This article was submitted to

Systems Endocrinology,

a section of the journal

Frontiers in Endocrinology

Received: 23 September 2021

Accepted: 08 December 2021

Published: 03 January 2022

Citation:

He Y, Zhu H, Zhang M, Li J, Ma S, $L u Y$, Chen $L$, Zhang $M$ and Peng $H$ (2022) Association Between Serum Furin and Fasting Glucose: A CrossSectional Study in Chinese Adults.

Front. Endocrinol. 12:781890. doi: 10.3389/fendo.2021.781890
Background: Furin has been associated with glucose metabolic phenotypes in small sampled clinical studies. However, this association has not yet been studied in Chinese. Here, we aimed to examine the association between serum furin and fasting glucose in Chinese adults.

Methods: Serum furin and fasting plasma glucose were assayed for 2,172 participants (mean aged 53 years, 38\% men) in the Gusu cohort. A median regression model was applied to examine the association between serum furin and fasting glucose, adjusting for age, sex, education level, cigarette smoking, alcohol drinking, obesity, blood pressure, and lipids. To facilitate data interpretation, the association between serum furin and prevalent diabetes was also examined.

Results: Serum furin was negatively associated with fasting glucose $(\beta=-0.18, P<0.001$ for log-furin). In participants with diabetes, serum furin was significantly lower than those with normal glucose (median: $0.90 \mathrm{ng} / \mathrm{mL}$ vs. $1.05 \mathrm{ng} / \mathrm{mL}, P=0.001$ ). Compared with participants in the highest quartile of serum furin, those in the lowest quartile had $42 \%$ and $80 \%$ increased risk of prevalent prediabetes $(\mathrm{OR}=1.42,95 \% \mathrm{Cl}: 1.05-1.92, P=0.023)$ and diabetes (OR=1.80, 95\%Cl: 1.13-2.91, $P=0.015)$, respectively.

Conclusions: Serum furin was negatively associated with prediabetes and diabetes in Chinese adults. Our findings suggest that serum furin may be a risk factor or a biomarker of diabetes.

Keywords: furin, diabetes, fasting plasma glucose, Chinese, cross-sectional study, risk factor

\section{INTRODUCTION}

Furin, ubiquitously expressed in all mammalian tissues and cells, is a member of the proprotein convertase subtilisin/Kexin (PCSK) family (1). Accumulating evidence has demonstrated that furin may be implicated in the process of glucose metabolism by converting numerous protein and peptide precursors into their bioactive forms. For example, cell-based studies found that furin could activate the precursors of insulin 
and its receptor $(2,3)$ and regulate the proliferation and differentiation of pancreatic $\beta$-cells which determines the secretion of insulin $(4,5)$. Animal experiments found that knockout of the furin gene in $\beta$-cells resulted in glucose intolerance in mice (6). In humans, polymorphisms in the FURIN gene have been associated with metabolic syndrome (7), hypertension (8), and coronary artery disease (9). These findings suggest a potential role of furin in glucose metabolism. In fact, the levels of furin in the circulation have been associated with diabetes (10) and some relative phenotypes, such as obesity (11), metabolic syndrome (12), and diabetic cardiovascular disease (13). However, these results were largely derived from populations with European ancestry. It's not clear whether circulating furin could be associated with glucose metabolism in Chinese who have a different genetic background and risk profiles of diabetes in comparison to European populations. In Chinese adults, we previously found that serum furin was associated with obesity (14) and hypertension (15). Whether serum furin is associated with diabetes has not been studied in Chinese. Therefore, we aimed to examine the association between serum furin and fasting plasma glucose in Chinese adults.

\section{METHODS}

\section{Participants}

The Gusu cohort is a prospective study aiming to identify new risk factors for cardiovascular disease (CVD) in Chinese adults. The study design, survey methods, and laboratory measurements have been detailed previously (16). In brief, 2,498 community members aged over 30 years and free of CVD and chronic kidney disease were enrolled in the baseline examination in 2010. After excluding participants with missing data on serum furin $(n=326), 2172$ participants were finally included in the current analysis. The protocols of the current study were approved by the Soochow University Ethics Committee (Approval No. SUDA20200601H02). Written informed consent was obtained from all study participants.

\section{Measurement of Serum Furin}

Using the $-80^{\circ} \mathrm{C}$ stored serums obtained in the baseline examination, furin concentrations were measured using commercial ELISA kits (Catalog: DL-FUR-Hu; DLDEVELOP, Wuxi, China) according to standard protocols as previously described (15). All the samples were processed in a duplicate assay. A standard curve was constructed and from which furin concentrations of unknown samples were determined.

\section{Measurement of Fasting Plasma Glucose}

Blood samples were obtained by venipuncture in the morning after a requested overnight fast (at least $8 \mathrm{~h}$ ). Within 4 hours after venipuncture, fasting plasma glucose (FPG) was analyzed enzymatically on a Hitachi 7020 automatic biochemical analyzer using commercial reagents (Kangxiang Medical Appliances, Shanghai, PR of China). Intra- and inter-assay coefficients of variation were less than $2 \%$ and $4 \%$, respectively. Diabetes was defined as the presence of one of the following: (a) a self-reported previous diagnosis by health care professionals and current use of either insulin or oral hypoglycemic medication, and (b) an FPG level of $7.0 \mathrm{mmol} / \mathrm{L}$ or higher (17). Prediabetes was defined as an FPG level between 5.6 and $6.9 \mathrm{mmol} / \mathrm{L}$ without self-reported physician-diagnosed diabetes.

\section{Assessment of Conventional Risk Factors}

Data on demographic information, lifestyle risk factors, and personal medical history were collected with standard questionnaires in the Chinese language administered by trained staff. Current smoking was defined as having smoked at least 100 cigarettes in the entire lifetime, smoke cigarettes regularly, and smoke currently. Current drinking was defined as having consumed alcohol $\geq 12$ times in the past year and drinking currently. Education level was estimated as years a participant stays in the education system. Body mass index (BMI) was calculated as weight in kilograms divided by the square of the subject's height in squared meters. Three consecutive sitting blood pressure measurements (3 min between each) were taken by trained staff, using a standard mercury sphygmomanometer, according to standard protocol (18), after the subjects had been resting for at least $5 \mathrm{~min}$. The first and fifth Korotkoff sounds were recorded as systolic blood pressure (SBP) and diastolic blood pressure (DBP), respectively. The mean of the three records was used in the analysis. Total cholesterol (TC), triglycerides (TG), high-density lipoprotein cholesterol (HDL-C), and low-density lipoprotein cholesterol (LDL-C) were examined for all participants.

\section{Statistical Analysis}

Baseline characteristics of study participants were presented according to quartiles of serum furin. Base-10 logarithmic transformation was applied to maximal normal distribution of serum furin and the generated values (log-furin) were used in downstream analyses. To examine the association between serum furin and FPG, we constructed a median regression model in which FPG was the dependent variable and serum furin (continuous logfurin or categorical furin in quartiles) was the independent variable, adjusting for age, sex, education level, cigarette smoking, alcohol drinking, BMI, SBP, LDL-C, and HDL-C. Median regression was used here to account for the skewed distribution data of FPG. To facilitate data interpretation, we further examined the association between serum furin and prevalent diabetes by constructing a logistic regression model in which prevalent diabetes (yes/no) was the dependent variable and serum furin (log-furin or furin quartiles) was the independent variable, adjusting for the confounding factors listed above. The association between serum furin and prediabetes was similarly examined. All statistical analyses were conducted using SAS statistical software (version 9.1, Cary, NC). A twotailed $P$ value less than 0.05 was considered statistically significant.

\section{RESULTS}

\section{Baseline Characteristics}

There were 2,172 participants (823 men and 1349 women) with a mean age of 53.2 years included in the present study. Of them, 
198 (9.12\%) participants including 85 patients under hypoglycemic treatments were diagnosed with prevalent diabetes. Their baseline characteristics according to quartiles of serum furin are shown in Table 1. Compared with participants with a higher level of serum furin, those with a lower level of serum furin were more likely to be older, current smokers, current drinkers and had higher levels of BMI, SBP, DBP, and FPG (all $P<0.05)$.

\section{Levels of Serum Furin in Participants With Different Statuses of Glucose Metabolism}

Intra- and inter-assay coefficients of variation of the measurements of serum furin were less than $10 \%$ and $12 \%$, respectively. Compared to participants with a normal FPG, the median levels of serum furin was significantly lower in those with prediabetes (median: $0.96 \mathrm{ng} / \mathrm{mL} v s .1 .05 \mathrm{ng} / \mathrm{mL}, P=0.003$ ) and diabetes (median: $0.90 \mathrm{ng} / \mathrm{mL}$ vs. $1.05 \mathrm{ng} / \mathrm{mL}, P=0.001$ ) (Figure 1). We did not find any significant difference in serum furin levels between participants with prediabetes and diabetes $(P=0.353)$.

\section{Association Between Serum Furin and Glucose Metabolic Status}

After adjusting for age, sex, education level, cigarette smoking, alcohol drinking, BMI, SBP, LDL-C, and HDL-C, serum furin was negatively associated with FPG $(\beta=-0.18, P<0.001$ for logfurin, Table 2). Compared with participants at the highest quartile of serum furin, those at the lowest quartile had a median of $0.19 \mathrm{mmol} / \mathrm{L}$ higher FPG $(\mathrm{P}<0.001)$. The regression with prevalent prediabetes and diabetes revealed that a higher level of serum furin seemed to be nominally associated with a lower risk of having prediabetes $(\mathrm{OR}=0.72, P=0.051)$ and diabetes $(\mathrm{OR}=0.62, P=0.052)$ (Table 3). Compared with participants at the highest quartile of serum furin, those at the lowest quartile had $42 \%$ and $80 \%$ increased risk of having prediabetes $(P=0.023)$ and diabetes $(P=0.015)$, respectively.

\section{DISCUSSION}

We are the first to report an association between serum furin and fasting glucose in Chinese adults in a cross-sectional study including a relatively large sample of participants in the Gusu cohort. Participants with a lower level of serum furin were more likely to have prevalent prediabetes and diabetes. This association was independent of behavioral and metabolic factors. Our findings indicated that furin may participate in glucose metabolism through mechanisms beyond metabolic factors. Furin deficiency may be a marker or even a potential risk factor for diabetes.

The identified association between serum furin and glucose metabolism found in our study has also been suggested by other studies. For example, $\beta$ cell-specific furin gene knockout mice developed glucose intolerance and had smaller islets with lower insulin content than wild controls (6). In humans, polymorphisms of the FURIN gene encoding furin protein have been associated with some diabetic complications, such as metabolic syndrome (7), hypertension (8), and coronary artery disease (9). Further, furin protein in circulation has also been studied by some clinical studies. For example, a case-control study including 25 diabetic patients with complications, 25 diabetic patients without complications, and 25 healthy controls found that serum furin was significantly associated with diabetes complicated with cardiovascular disease (13). A cross-sectional study including 138 participants found that furin was significantly associated with metabolic syndrome (12). A prospective study including 4,678 participants reported an association of serum furin with incident diabetes among Swedish in the Malmö Diet and Cancer Study (10). However, these results were mainly generated from White populations whose genetic background and diabetes risk profiles differ from Chinese. Our study is the first to examine the association between serum furin and diabetes in Chinese adults. Nevertheless, we found an inconsistent result that a lower level

TABLE 1 | Baseline characteristics of study participants according to serum furin levels ( $N=2172)$.

\begin{tabular}{|c|c|c|c|c|c|}
\hline \multirow[t]{2}{*}{ Characteristics } & \multicolumn{4}{|c|}{ Serum furin, $\mathrm{ng} / \mathrm{mL}$} & \multirow[t]{2}{*}{$P$-value for trend } \\
\hline & Quartile 1 ( 0.59) & Quartile 2 (0.60 1.01) & Quartile 3 (1.02 1.75) & Quartile 4 (1.76 ) & \\
\hline No. of participants & 544 & 543 & 543 & 542 & \\
\hline Age, years, mean $\pm S D$ & $54.37 \pm 9.51$ & $53.49 \pm 9.51$ & $51.90 \pm 9.52$ & $51.58 \pm 9.57$ & $<0.001$ \\
\hline Sex, men (\%) & 254 (46.69) & $208(38.31)$ & $180(33.15)$ & 181 (33.39) & $<0.001$ \\
\hline Current smoking, n (\%) & $146(26.84)$ & $122(22.47)$ & $96(17.68)$ & $121(22.32)$ & 0.022 \\
\hline Current drinking, $\mathrm{n}(\%)$ & $128(23.53)$ & $103(18.97)$ & $88(16.21)$ & $76(14.02)$ & $<0.001$ \\
\hline Education level, years, mean \pm SD & $7.18 \pm 3.31$ & $7.32 \pm 3.14$ & $7.04 \pm 3.26$ & $6.83 \pm 3.21$ & 0.033 \\
\hline $\mathrm{BMl}, \mathrm{kg} / \mathrm{m}^{2}$, mean $\pm \mathrm{SD}$ & $24.91 \pm 3.70$ & $25.10 \pm 3.46$ & $24.78 \pm 3.46$ & $24.51 \pm 3.83$ & 0.031 \\
\hline $\mathrm{SBP}, \mathrm{mmHg}$, mean $\pm \mathrm{SD}$ & $132.9 \pm 15.5$ & $131.1 \pm 14.3$ & $129.1 \pm 15.8$ & $128.3 \pm 20.1$ & $<0.001$ \\
\hline $\mathrm{DBP}, \mathrm{mmHg}$, mean $\pm \mathrm{SD}$ & $86.3 \pm 7.6$ & $85.6 \pm 8.1$ & $84.4 \pm 8.9$ & $84.0 \pm 11.3$ & $<0.001$ \\
\hline Fasting glucose, mmol/L & $5.3(4.8-5.8)$ & $5.2(4.7-5.7)$ & $5.1(4.7-5.6)$ & $5.0(4.6-5.6)$ & 0.002 \\
\hline Total cholesterol, mmol/L & 5.05 (4.50-5.63) & $5.10(4.56-5.74)$ & $5.09(4.53-5.77)$ & $5.11(4.55-5.68)$ & 0.154 \\
\hline Triglycerides, mmol/L & $1.09(0.79-1.50)$ & $1.19(0.82-1.70)$ & $1.08(0.77-1.68)$ & $1.11(0.75-1.65)$ & 0.428 \\
\hline LDL cholesterol, mmol/L & $2.96(2.56-3.50)$ & $2.97(2.51-3.46)$ & $2.93(2.50-3.37)$ & $2.92(2.48-3.39)$ & 0.164 \\
\hline HDL cholesterol, mmol/L & $1.44(1.24-1.71)$ & $1.43(1.19-1.70)$ & $1.50(1.26-1.75)$ & $1.45(1.22-1.72)$ & 0.612 \\
\hline
\end{tabular}

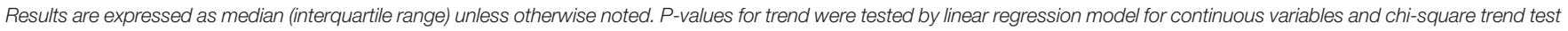
for categorical variables.

BMI, body mass index; SBP, systolic blood pressure; DBP, diastolic blood pressure; LDL, low-density lipoprotein; HDL, high-density lipoprotein. 


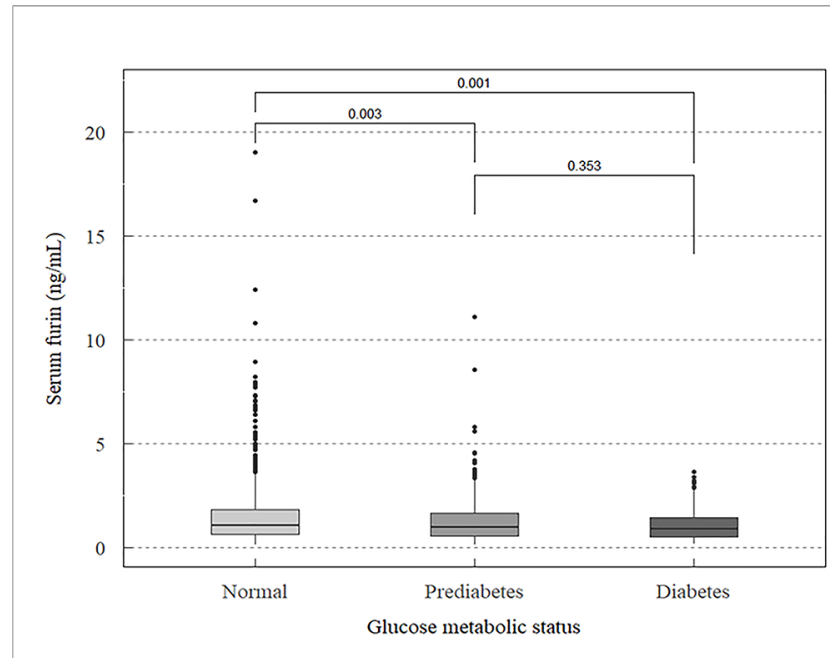

FIGURE 1 | Median levels of serum furin in participants with different statuses of glucose metabolism.

of serum furin was associated with a higher risk of prevalent diabetes. Different genetic backgrounds of study participants may explain the discrepancy. Notably, basic studies demonstrated that furin deficiency caused by gene knockout could result in glucose intolerance (6). This finding may indicate a feedback adjustment mechanism that increased glucose in diabetic patients stimulates secretion of furin in feedback to facilitate activation of insulin and its receptors. Therefore, researchers may observe a positive association between serum furin and diabetes in populations as listed above. These results appeared to indicate that the relation between serum furin and diabetes in populations is more complex than that in cell and animal experiments. As a wide proprotein convertase, furin acts as an upstream regulator of the glucose metabolism system, such as the natriuretic peptides system. This system plays a critical role in maintaining blood glucose balance and blood pressure through BNP which was activated by furin. As a result, furin expression and excretion may be upregulated in compensatory for high-risk individuals of diabetes, e.g., hypertensive and obesity patients. Also, our group previously found that serum furin was also significantly associated with obesity and hypertension that share many risk factors and molecular mechanisms with diabetes $(14,15)$. All these findings, even conflicting, together with ours, suggest that furin may participate in glucose metabolism and therefore could be a therapeutic target for diabetes.

In addition to population evidence, the possible mechanisms underlying the association between serum furin and diabetes could deepen our understanding of the role of furin in diabetes. Furin, which belongs to the PCSK family and converts numerous proteins and peptide precursors into their biologically active forms, has been demonstrated to play an important role in glucose metabolism and several relative processes associated with diabetes, such as insulin resistance. One possible mechanism could be that furin deficiency influences the maturation of insulin receptors $(2,19)$ which is critical for the maintenance of glucose homeostasis. The second possible mechanism may be the involvement of furin in the proliferation and differentiation of pancreatic $\beta$-cells (5) where glucose metabolism mainly occurs. Another possible mechanism might be related to the role of furin in the activation of B-type natriuretic peptides $(20,21)$ which is one key component of the natriuretic peptides system. This system has been demonstrated to play an integral role in glucose metabolism and participate in the development of diabetes $(20,22,23)$.

TABLE 2 | Association between serum furin and fasting plasma glucose.

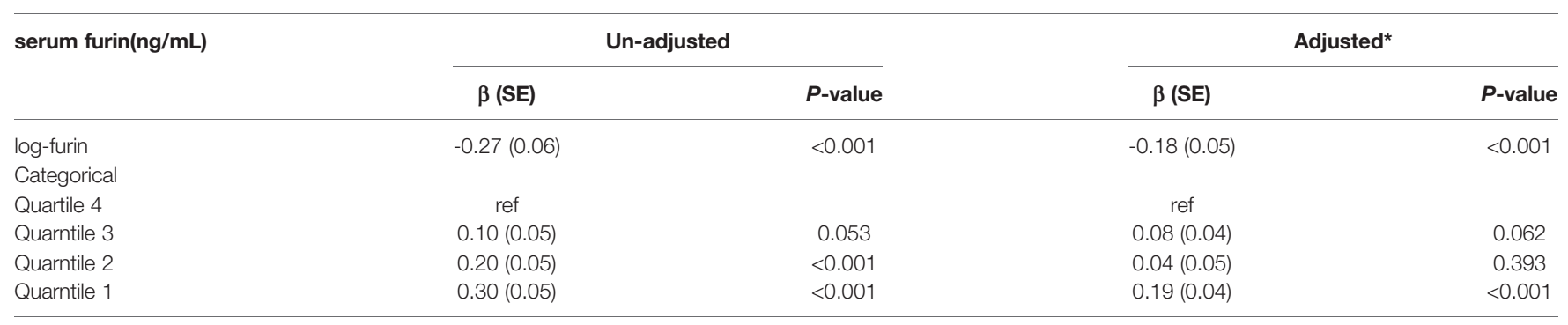

*Adjusting for age, sex, education level, cigarette smoking, alcohol consumption, SBP, BMI, LDL, and HDL.

TABLE 3 | Associations between serum furin and prevalent prediabetes and diabetes.

\begin{tabular}{|c|c|c|c|c|c|c|}
\hline serum furin(ng/mL) & \multicolumn{3}{|c|}{ Prediabetes } & \multicolumn{3}{|c|}{ Diabetes } \\
\hline $\begin{array}{l}\text { log-furin } \\
\text { Categorical }\end{array}$ & 474 & $0.72(0.52-1.00)$ & 0.051 & 198 & $0.62(0.38-1.00)$ & 0.052 \\
\hline Quartile 4 & 108 & ref & & 33 & ref & \\
\hline Quartile 3 & 107 & $1.05(0.76-1.43)$ & 0.779 & 55 & $1.74(1.08-2.82)$ & 0.024 \\
\hline Quartile 2 & 114 & $0.98(0.72-1.34)$ & 0.910 & 48 & $1.28(0.79-2.10)$ & 0.315 \\
\hline
\end{tabular}

${ }^{*}$ Adjusting for age, sex, education level, cigarette smoking, alcohol consumption, SBP, BMI, LDL, and HDL. 
To the best of our knowledge, our study is the first to investigate the association between serum furin and diabetes in Chinese adults. The strengths of this study include careful and systemic analyses of the association between serum furin and glucose metabolic states including prediabetes and diabetes and comprehensive adjustments of many conventional risk factors including behavioral and metabolic factors. However, our study also has several limitations that deserve clarification. First, the cross-sectional study designs prevented a causal inference. However, our results showed that serum furin was not only associated with diabetes but also correlated with prediabetes. This finding increased the probability that furin deficiency may be a risk factor for diabetes. Nevertheless, the causality between serum furin and diabetes is not established and needs further evidence from clinical trials. Second, our study population was comprised of ethnic Han individuals only, whether the results can be generalizable to other racial/ethnic groups is not clear.

\section{CONCLUSION}

In conclusion, our study demonstrated that serum furin was negatively associated with prediabetes and diabetes in Chinese adults. These results suggested that furin might play a potential role in glucose metabolism and the deficiency of furin might serve as a risk factor or biomarker for diabetes. However, the causal association between furin and diabetes still needs more evidence and the underlying molecular mechanisms still need further investigations.

\section{DATA AVAILABILITY STATEMENT}

The raw data supporting the conclusions of this article will be made available by the authors, without undue reservation.

\section{REFERENCES}

1. Seidah NG, Day R, Marcinkiewicz M, Chrétien M. Precursor Convertases: An Evolutionary Ancient, Cell-Specific, Combinatorial Mechanism Yielding Diverse Bioactive Peptides and Proteins. Ann N Y Acad Sci (1998) 839:924. doi: 10.1111/j.1749-6632.1998.tb10727.x

2. Bravo DA, Gleason JB, Sanchez RI, Roth RA, Fuller RS. Accurate and Efficient Cleavage of the Human Insulin Proreceptor by the Human ProproteinProcessing Protease Furin. Characterization and Kinetic Parameters Using the Purified, Secreted Soluble Protease Expressed by a Recombinant Baculovirus. J Biol Chem (1994) 269:25830-7. doi: 10.1016/S0021-9258(18) 47322-X

3. Vollenweider F, Kaufmann J, Irminger JC, Halban PA. Processing of Proinsulin by Furin, PC2, and PC3 in (Co) Transfected COS (Monkey Kidney) Cells. Diabetes (1995) 44:1075-80. doi: 10.2337/diab.44.9.1075

4. Louagie E, Taylor NA, Flamez D, Roebroek AJ, Bright NA, Meulemans S, et al. Role of Furin in Granular Acidification in the Endocrine Pancreas: Identification of the V-ATPase Subunit Ac45 as a Candidate Substrate. Proc Natl Acad Sci USA (2008) 105:12319-24. doi: 10.1073/pnas.0800340105

5. Kayo T, Sawada Y, Suda M, Konda Y, Izumi T, Tanaka S, et al. ProproteinProcessing Endoprotease Furin Controls Growth of Pancreatic Beta-Cells. Diabetes (1997) 46:1296-304. doi: 10.2337/diabetes.46.8.1296

\section{ETHICS STATEMENT}

The studies involving human participants were reviewed and approved by Soochow University Ethics Committee. The patients/participants provided their written informed consent to participate in this study.

\section{AUTHOR CONTRIBUTIONS}

$\mathrm{YH}, \mathrm{MZZ}$, and HP conceived and designed the study. $\mathrm{HZ}$ and $\mathrm{MZ}$ analyzed and interpreted the data. $\mathrm{YH}$ and $\mathrm{HZ}$ drafted the paper. MZ, YH, JL, YL, LC, and SM collected the data. MZZ and $\mathrm{HP}$ revised and gave the final approval of the version to be published, and all authors agreed to be accountable for all aspects of the work.

\section{FUNDING}

This study was supported by the National Natural Science Foundation of China (NO. 82173596, 81903384, and 81872690 ), the Natural Science Foundation of Jiangsu Province (NO. BK20180841), the Suzhou Municipal Science and Technology Bureau (NO. SS201853, SKJY2021040, and SYS2020091), and a Project of the Priority Academic Program Development of Jiangsu Higher Education Institutions.

\section{ACKNOWLEDGMENTS}

We are deeply appreciative of the cooperation and participation of the members in the Gusu cohort and thank all staff for their support and assistance. Especially, we thank the Gusu Center for Disease Prevention and Control for their support in the recruitment of participants.

6. Brouwers B, Coppola I, Vints K, Dislich B, Jouvet N, Van Lommel L, et al. Loss of Furin in $\beta$-Cells Induces an Mtorc1-ATF4 Anabolic Pathway That Leads to $\beta$-Cell Dysfunction. Diabetes (2021) 70:492-503. doi: 10.2337/db200474

7. Ueyama C, Horibe H, Yamase Y, Fujimaki T, Oguri M, Kato K, et al. Association of FURIN and ZPR1 Polymorphisms With Metabolic Syndrome. Biomed Rep (2015) 3:641-7. doi: 10.3892/br.2015.484

8. Ehret GB, Munroe PB, Rice KM, Bochud M, Johnson AD, Chasman DI, et al. Genetic Variants in Novel Pathways Influence Blood Pressure and Cardiovascular Disease Risk. Nature (2011) 478:103-9. doi: 10.1038/ nature 10405

9. Deloukas P, Kanoni S, Willenborg C, Farrall M, Assimes TL, Thompson JR, et al. Large-Scale Association Analysis Identifies New Risk Loci for Coronary Artery Disease. Nat Genet (2013) 45:25-33. doi: 10.1038/ ng. 2480

10. Fernandez C, Rysä J, Almgren P, Nilsson J, Engström G, Orho-Melander M, et al. Plasma Levels of the Proprotein Convertase Furin and Incidence of Diabetes and Mortality. J Internal Med (2018) 284:377-87. doi: 10.1111/ joim. 12783

11. Swärd P, Rosengren BE, Jehpsson L, Karlsson MK. Association Between Circulating Furin Levels, Obesity and Pro-Inflammatory Markers in Children. Acta Paediatr (Oslo Norway 1992) (2021) 110:1863-8. doi: 10.1111/apa.15774 
12. Harlid S, Myte R, Van Guelpen B. The Metabolic Syndrome, Inflammation, and Colorectal Cancer Risk: An Evaluation of Large Panels of Plasma Protein Markers Using Repeated, Prediagnostic Samples. Mediators Inflamm (2017) 2017:4803156. doi: 10.1155/2017/4803156

13. Fathy SA, Abdel Hamid FF, Zabut BM, Jamee AF, Ali MA, Abu Mustafa AM. Diagnostic Utility of BNP, Corin and Furin as Biomarkers for Cardiovascular Complications in Type 2 Diabetes Mellitus Patients. Biomarkers (2015) 20:460-9. doi: 10.3109/1354750X.2015.1093032

14. He Y, Ren L, Zhang Q, Zhang M, Shi J, Hu W, et al. Deficient Serum Furin Predicts Risk of Abdominal Obesity: Findings From a Prospective Cohort of Chinese Adults. Postgrad Med J (2021) 97:234-8. doi: 10.1136/postgradmedj-2019-137422

15. He Y, Ren L, Zhang Q, Zhang M, Shi J, Hu W, et al. Serum Furin as a Biomarker of High Blood Pressure: Findings From a Longitudinal Study in Chinese Adults. Hypertens Res (2019) 42:1808-15. doi: 10.1038/s41440-019-0295-6

16. Peng H, Zhang Q, Cai X, Liu Y, Ding J, Tian H, et al. Association Between High Serum Soluble Corin and Hypertension: A Cross-Sectional Study in a General Population of China. Am J Hypertens (2015) 28:1141-9. doi: 10.1093/ajh/hpv002

17. American Dibetes Association. 2. Classification and Diagnosis of Diabetes: Standards of Medical Care in Diabetes-2020. Diabetes Care (2020) 43:S14s31. doi: 10.2337/dc20-S002

18. Weber MA, Schiffrin EL, White WB, Mann S, Lindholm LH, Kenerson JG, et al. Clinical Practice Guidelines for the Management of Hypertension in the Community: A Statement by the American Society of Hypertension and the International Society of Hypertension. J Clin Hypertens (Greenwich Conn) (2014) 16:14-26. doi: 10.1111/jch.12237

19. Bernot D, Stalin J, Stocker P, Bonardo B, Scroyen I, Alessi MC, et al. Plasminogen Activator Inhibitor 1 is an Intracellular Inhibitor of Furin Proprotein Convertase. J Cell Sci (2011) 124:1224-30. doi: 10.1242/jcs.079889

20. Verboven K, Hansen D, Jocken JWE, Blaak EE. Natriuretic Peptides in the Control of Lipid Metabolism and Insulin Sensitivity. Obes Rev (2017) 18:1243-59. doi: 10.1111/obr.12598
21. Semenov AG, Tamm NN, Seferian KR, Postnikov AB, Karpova NS, Serebryanaya DV, et al. Processing of Pro-B-Type Natriuretic Peptide: Furin and Corin as Candidate Convertases. Clin Chem (2010) 56:1166-76. doi: 10.1373 /clinchem.2010.143883

22. Pfister R, Sharp S, Luben R, Welsh P, Barroso I, Salomaa V, et al. Mendelian Randomization Study of B-Type Natriuretic Peptide and Type 2 Diabetes: Evidence of Causal Association From Population Studies. PloS Med (2011) 8: e1001112. doi: 10.1371/journal.pmed.1001112

23. Heinisch BB, Vila G, Resl M, Riedl M, Dieplinger B, Mueller T, et al. BType Natriuretic Peptide (BNP) Affects the Initial Response to Intravenous Glucose: A Randomized Placebo-Controlled Cross-Over Study in Healthy Men. Diabetologia (2012) 55:1400-5. doi: 10.1007/ s00125-011-2392-1

Conflict of Interest: The authors declare that the research was conducted in the absence of any commercial or financial relationships that could be construed as a potential conflict of interest.

Publisher's Note: All claims expressed in this article are solely those of the authors and do not necessarily represent those of their affiliated organizations, or those of the publisher, the editors and the reviewers. Any product that may be evaluated in this article, or claim that may be made by its manufacturer, is not guaranteed or endorsed by the publisher.

Copyright (c) $2022 \mathrm{He}$, Zhu, Zhang, Li, Ma, Lu, Chen, Zhang and Peng. This is an open-access article distributed under the terms of the Creative Commons Attribution License (CC BY). The use, distribution or reproduction in other forums is permitted, provided the original author(s) and the copyright owner(s) are credited and that the original publication in this journal is cited, in accordance with accepted academic practice. No use, distribution or reproduction is permitted which does not comply with these terms. 